\title{
Exploiting the Unusual to Find the Rare: Novel Steroid Metabolites Pinpoint Adrenal Cancer Patients
}

\author{
Tobias Else $^{1} \cdot$ Richard J. Auchus $^{1} \cdot$ William E. Rainey ${ }^{1,2}$
}

Received: 1 June 2015 / Accepted: 1 June 2015 / Published online: 12 June 2015

(C) Springer Science+Business Media New York 2015

Half a century ago, endocrinology practice was limited to patients with advanced, overt disease. The advent of routine clinical laboratory testing spawned the recognition of early and subclinical disease, which has dominated endocrine practice in recent decades. Today, clinical endocrinology faces a new challenge of potential occult disease presenting from incidental findings. One such challenge is the rising number of patients diagnosed with incidental adrenal tumors as a result of cross-sectional imaging procedures unrelated to the evaluation of the adrenal glands. This quandary reverses the traditional endocrine dogma of biochemical diagnosis before imaging and obligates the exclusion of morbid pathology in an asymptomatic patient. The evaluation of patients with adrenal tumors for overt hormone production through targeted hormonal analysis is well established in endocrine care. In contrast, the presence of an adrenal tumor challenges clinicians to exclude the diagnosis of the very rare but deadly adrenal cancers (incidence 1/million) amongst the common adrenal nodules (prevalence up to $5 \%$ ). Finding the adrenal cancer needle in the haystack of adrenal nodules is critical because early diagnosis is the best hope for cure by surgery [1].

Currently, the differentiation of malignant vs. benign adrenal tumors is largely based on imaging characteristics. However, a full imaging work-up involves high cost (MRI),

Tobias Else

telse@umich.edu

1 Division of Metabolism, Endocrinology and Diabetes (MEND), Department of Internal Medicine, University of Michigan Health System, 2560E MSRB2, 1150 West Medical Center Drive, Ann Arbor, MI 48109-5674, USA

2 Department of Molecular and Integrative Physiology, University of Michigan, Ann Arbor, MI, USA radiation exposure (CT and $\mathrm{PET}$ ), and residual uncertainty with at least 5-10\% of adrenal tumors in an indeterminate category. In addition, imaging surveillance of all indeterminate lesions is of unproven overall benefit, and it is likely that several malignant lesions might still escape diagnosis in the recommended surveillance time frame (with regards to this question also see the article in this issue by Nogueira and Lirov et al. [2]). Therefore, other methods that can identify patients with adrenal cancers are desperately needed. In this issue of Hormones and Cancer, Kerkhofs et al. describe the use of a method at the very core of endocrine practice, the measurement of hormones and hormone metabolites, hypothesizing that most adrenocortical carcinomas (ACCs) produce unusual steroids and steroid metabolites that can be exploited for the diagnosis of the disease [3].

However, this approach requires an analytical method that measures multiple steroids at the same time with high specificity and sensitivity, overcoming the shortcomings of traditional immunoassays, which are limited to measuring one steroid at a time, often with limited specificity (due to crossreactivity). Over the course of the last decades, mass spectrometry (MS)-based analytic systems, such as GC/MS and LC-MS/MS, have become more available and competitive, allowing their translation into routine clinical care. With regard to steroid hormones, MS-based analytics allow for the measurement of multiple steroids in the same run from one sample.

Kerkhofs et al. studied a cohort of patients with incidental adrenal masses and adrenal cancers analyzing urine steroid metabolites and steroid hormones by GC/MS. They find some steroids, which provide excellent discrimination of ACC from any of the other lesions. Interestingly, these metabolites are ordinarily minor products and not active adrenal hormones. The biomarkers of ACC are derived from steroid intermediates, which are not normally measured in the endocrine 
evaluation of an incidental adrenal nodule. Although the idea is not entirely new, the current study certainly brings MSbased analytics one step closer to routine endocrine care. As early as 1954, increased excretion of tetrahydro-11deoxycortisol (THS), the major 11-deoxycortisol metabolite, was found to be disproportionately elevated in the urine of an ACC patient, and this finding was confirmed in several smaller studies [4-6]. The basis of the increase of 11-deoxycortisol metabolites is likely the relative deficiency of the enzyme $11 \beta$-hydroxylase (CYP11B1), which has significantly lower expression in ACC when compared to benign tumors [7]. In 2011, the European Network for the Study of Adrenal Tumors (ENS@T,www.ensat.org) demonstrated that THS was significantly elevated in the urine of 45 ACC patients compared to urine from 102 adrenocortical adenoma patients [8]. The ENS@T group also found elevated amounts of steroid precursor metabolites 5-pregnenediol and 5pregnentriol - the metabolites of pregnenolone and 17hydroxypregnenolone, respectively - in urine from ACC patients. Using their data, they developed a sophisticated mathematical algorithm for predicting the chances that an adrenal tumor is an ACC from urine steroid profiling. Now, Kerkhofs et al. have replicated the results of the ENS@T group, leaving little doubt that urine steroid profiling is a highly specific and quite sensitive method to diagnose ACC preoperatively.

What prevents routine incorporation of urine steroid profiling in the management of patients with incidental adrenal tumors in general and ACC in particular? First is assay availability. The cost of the GC/MS instrumentation is not generally prohibitive, $<\$ 100,000$, but expertise in this technique is not typical in clinical laboratories. The urine steroid profiling is not a simple "kit" with detailed instructions but rather a homemade product, which requires significant method development effort, even starting with a published protocol. Sample preparation and derivatization is tedious, taking 2-3 days, and scrupulous attention to quality controls and internal standards are essential. Pitfalls and troubleshooting skills are only acquired with time. All of these considerations create hesitation for setting up the assay, but none of these hurdles are insurmountable, as many laboratories around the world have shown. Is cost a limitation? It is hard to imagine a urine assay costing more than repeated imaging, clinic visits, and blood testing. We believe that demand will drive reference laboratories to offer these methods not as a boutique curiosity limited to research studies but as mainstream medical practice. Finally, if the urine metabolites are characteristically elevated, the corresponding steroids produced from the tumors themselves might be easy targets for serum measurements. Furthermore,
LC-MS/MS methods to profile serum steroids are well developed with much quicker turn-around times than GC/MS profiling [9].

Finally, the time has come to apply the urine steroid profiling strategy in a prospective fashion to the large patient population with adrenal tumors. Of course, the challenge is the overall rarity of ACC, which can only be overcome with collaborative multicenter trials and after exclusion of obviously benign tumors by the established imaging procedures to increase the number of adrenal tumors that have a higher likelihood of malignancy. Going forward, the results presented by Kerkhofs et al. also pave the way for a broader applicability of MS measured steroid metabolites as a tool to investigate cancer recurrence and tumor burden in ACC patients.

Conflict of Interest R.J. Auchus is a consultant for Laboratory Corporation of America of America.

W.E. Rainey is a member of the Scientific Advisory Board for Atterocor

T. Else has nothing to disclose.

\section{References}

1. Else T, Kim AC, Sabolch A et al (2014) Adrenocortical carcinoma. Endocr Rev 35:282-326

2. Nogueira TL R, Caoili EM, Lerario AM, Miller BS, Fragoso MCBV, Dunnick NR, Hammer GD, Else T (2015) Radiographic characteristics of incidentally discovered masses in patients later diagnosed with adrenocortical cancer. Horm Cancerormones Cancer

3. Kerkhofs TK, MN, Kema IP, Willems TP, Haak HR (2015) Diagnostic value of urinary steroid profiling in the evaluation of adrenal tumors. Horm Cancer

4. Grondal S, Eriksson B, Hagenas L, Werner S, Curstedt T (1990) Steroid profile in urine: a useful tool in the diagnosis and follow up of adrenocortical carcinoma. Acta Endocrinol 122:656-663

5. Minowada S, Kinoshita K, Hara M, Isurugi K, Uchikawa T, Niijima $\mathrm{T}$ (1985) Measurement of urinary steroid profile in patients with adrenal tumor as a screening method for carcinoma. Endocrinol Jpn 32:29-37

6. Touchstone JC, Richardson EM, Bulaschenko H, Landolt I, Dohan FC (1954) Isolation of pregnane-3-alpha, 17-alpha, 21-triol-20-one (tetrahydro compound $\mathrm{S}$ ) from the urine of a woman with metastatic adrenocortical carcinoma. J Clin Endocrinol Metab 14:676-678

7. Giordano TJ, Kuick R, Else T et al (2009) Molecular classification and prognostication of adrenocortical tumors by transcriptome profiling. Clin Cancer Res Off J Am Assoc Cancer Res 15:668-676

8. Arlt W, Biehl M, Taylor AE et al (2011) Urine steroid metabolomics as a biomarker tool for detecting malignancy in adrenal tumors. $\mathrm{J}$ Clin Endocrinol Metab 96:3775-3784

9. Turcu AF, Rege J, Chomic R, et al. (2015) Profiles of 21-carbon steroids in 21-hydroxylase deficiency. J Clin Endocrinol Metab jc20151023 